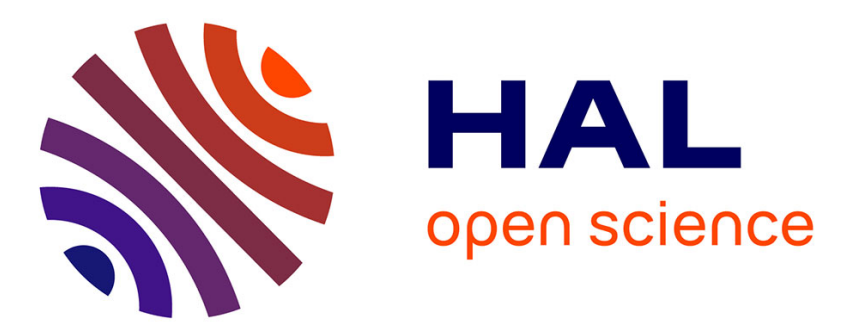

\title{
RaDMaX: a graphical program for the determination of strain and damage profiles in irradiated crystals
}

\author{
M. Souilah, Alexandre Boulle, A. Debelle
}

\section{To cite this version:}

M. Souilah, Alexandre Boulle, A. Debelle. RaDMaX: a graphical program for the determination of strain and damage profiles in irradiated crystals. Journal of Applied Crystallography, 2016, 49 (1), pp.311-316. 10.1107/S1600576715021019 . hal-02193176

\section{HAL Id: hal-02193176 \\ https://hal.science/hal-02193176}

Submitted on 24 Jul 2019

HAL is a multi-disciplinary open access archive for the deposit and dissemination of scientific research documents, whether they are published or not. The documents may come from teaching and research institutions in France or abroad, or from public or private research centers.
L'archive ouverte pluridisciplinaire HAL, est destinée au dépôt et à la diffusion de documents scientifiques de niveau recherche, publiés ou non, émanant des établissements d'enseignement et de recherche français ou étrangers, des laboratoires publics ou privés. 


\title{
RaDMaX: a graphical program for the determination of strain and damage profiles in irradiated crystals
}

\author{
M. Souilah ${ }^{\text {a }}$, A. Boulle ${ }^{\text {a }}$, A. Debelle ${ }^{b}$ \\ ${ }^{a}$ Science des Procédés Céramiques et Traitements de Surfaces, CNRS UMR 7315, Centre Européen \\ de la Céramique, 12 rue Atlantis, 87068 Limoges Cedex, France. \\ ${ }^{\mathrm{b}}$ Centre de Sciences Nucléaires et de Sciences de la Matière, Université Paris-Sud, CNRS/IN2P3, \\ 91405 Orsay, France.
}

\begin{abstract}
RaDMaX (Radiation Damage in Materials analyzed with X-ray diffraction ) is a user-friendly graphical program that allows to determine the strain and damage depth profiles in ion-irradiated crystals. This task is achieved by fitting experimental X-ray diffraction data, recorded in symmetrical $\theta-2 \theta$ geometry, with a dynamical diffraction model parametrized with variable strain and damage profiles based on $B$-spline functions. The strain and damage profiles can be graphically manipulated so as to fit the calculated curve to the experimental data. Automatic fitting procedures (generalized simulated annealing and conventional least-squares) are also implemented. RaDMaX is free and open-source (CeCILL license) and can be downloaded from: http://aboulle.github.io/RaDMaX.
\end{abstract}

\section{Introduction}

Ion beams produced by particle accelerators are used in many fields of materials science, for instance to simulate radiative environments (Thomé \& Garrido, 2001), for the implantation of semiconductor materials (Elliman \& Williams, 2015) or to synthesize nanostructured materials 
(Dhara, 2007). The collision of energetic ions with the atoms of the target material produces crystalline damage which, in the first irradiation stages, usually consists in the target atoms being displaced from their equilibrium positions and formation of Frenkel pairs (i.e. a target atom is transferred from its nominal site to an interstitial position, leaving behind a vacancy). With increasing irradiation dose, the initial point defects may further rearrange to form larger scale defects like point-defect clusters, dislocation loops or may even lead to a full amorphization of the material (Averback, 1994; Weber, 2000).

X-ray diffraction (XRD) is extremely sensitive to atomic displacements and is used to study radiation damage in single crystals since several decades (Speriosu, 1981; Zaumseil et al., 1987; Klappe \& Fewster, 1994; Milita \& Servidori, 1995; Boulle \& Debelle, 2010; Rieutord et al., 2013). From a phenomenological standpoint, despite the underlying structural complexity of irradiated materials, radiation damage gives rise to two simple distinct features when considering the coherent X-ray diffraction signal: strain, yielding a shift of the Bragg peaks, and random lattice displacements, leading to a lowering of the coherent intensity (and to the concomitant increase of diffuse scattering intensity). The strain and lattice displacements (hereinafter simply referred to as "damage") are in general inhomogeneously distributed within a subsurface region with thickness ranging from a few nanometers to several tens of microns, depending on the energy of the incident ions. Whether ion beams are used to intentionally modify the materials, or to simulate their degradation under irradiation, a detailed knowledge of the strain and damage profiles in irradiated materials is essential, as those might profoundly affect their properties. Additionally, the determination of strain and damage profiles is often also a necessary "first-step" before a more indepth analysis of the structure of irradiated materials.

Because irradiated single crystals actually resemble a simple film/substrate system the associated XRD data can be analyzed using formalisms developed for the study of epitaxial films and heterostructures (Pietsch et al., 2004). There are numerous, either freely available or commercial, 
simulation packages that have been developed to this end. One particularly popular program is Sergey Stepanov's X-ray server (http://sergey.gmca.aps.anl.gov/) which is a web-based graphical interface that allows to simulate the XRD intensity distribution in a variety of scattering geometries. We here present a new program devoted to the analysis of radiation damage in crystals: RaDMaX (Radiation Damage in Materials analyzed with XRD). In contrast to existing solutions, RaDMaX has been developed to perform just one single task, i.e. the determination of strain and damage profiles in irradiated crystals, and to make this task as simple and fast as possible. The program is accessible to users with only little crystallographic knowledge, although the source code is open and advanced users can use it to adapt it to particular needs. Central to RaDMaX is the existence of interactive plots of the strain and damage profiles which allow the users to graphically modify the profiles and have the corresponding XRD curve computed and updated in real time. Once a roughly acceptable solution has been obtained, either graphically and/or via the import of strain/damage profiles obtained by complementary techniques, automatic procedures can be used to fit the calculated curve to experimental data, and in turn obtain the corresponding strain/damage profiles.

\section{Features and implementation}

In this section we briefly review the principles underlying the functioning of the program. Detailed mathematical information can be found in our previous publications (Boulle et al., 2003; Boulle et al., 2009, Boulle \& Debelle, 2010). In RaDMaX, the determination of strain/damage profiles is achieved by fitting a computed XRD curve to experimental XRD data, and refining the strain/damage profiles until the best possible agreement is reached. The calculation of the XRD curves is performed within the framework of the dynamical theory of diffraction from distorted crystals (Takagi, 1969; Taupin, 1964) which has proven to be particularly relevant for the study of irradiated materials (Klappe \& Fewster, 1994; Milita \& Servidori, 1995; Boulle \& Debelle, 2010), especially considering the strong diffraction from single crystals. In particular, we make use of the 
recursive solution to the Takagi-Taupin equations proposed by Bartels, Hornstra \& Lobeek (1986). In this approach the irradiated region is divided into $N_{l}$ lamellas where all structural properties (strain, damage, structure factor, etc.) are constant. Starting from the scattering at the film/substrate interface, which corresponds to the scattering from the undistorted bulk material, the total scattered amplitude is constructed iteratively by combining the amplitudes scattered and transmitted at each interface, up to the surface. The critical parameter in this approach is the number of lamellas which, for accuracy, has to be as high as possible, but not too high in order not to slow down the calculations. Guidelines for optimizing this parameter will be given in the next section.

The values of strain and damage within each lamella have to be progressively adjusted in order to match the computed curve to the experimental data. The number of lamellas being in general relatively large (typically 50-100 for both the strain and the damage profiles), this task might be particularly cumbersome and the results of an automatic fitting procedure might lead to unphysical (oscillating or discontinuous) solutions. In order to stabilize the solution, we model the strain/damage profiles with $B$-spline functions (Boulle et al., 2003):

$$
\begin{aligned}
& e(z)=\sum_{i=1}^{N_{w}^{e}} w_{i}^{e} B_{i, 3}(z) \\
& D W(z)=\sum_{i=1}^{N_{w}^{D W}} w_{i}^{D W} B_{i, 3}(z)
\end{aligned}
$$

where $e(z)$ and $D W(z)$ are the strain and damage as a function of the depth below the surface, $z$. $B_{i, 3}(z)$ is the $B$-spline basis function of third degree and $w_{i}^{e}\left(w_{i}^{D W}\right)$ is the weight given to each basis function in the strain (damage) profile. $N_{w}{ }^{e}\left(N_{w}{ }^{D W}\right)$ is the total number of basis functions used for the strain (damage) profile. Typical values for $N_{w}{ }^{e}$ and $N_{w}{ }^{D W}$ are comprised between 5 and 15 . Guidelines for optimizing these parameters will be given in the next section. The static DebyeWaller (DW) factor is commonly used as a measure of lattice damage (Speriosu, 1981). The DW factor lowers the amplitude scattered from each lamella and is bounded between 1 (perfect crystal) and 0 (fully damaged crystal) (Boulle et al., 2005): 


$$
D W(z)=\langle\exp [i \mathbf{Q} \mathbf{u}(z)]\rangle
$$

where $\mathbf{u}(z)$ is the lattice displacement at depth $z, \mathbf{Q}$ is the scattering vector and the angular brackets indicate a spatial averaging over the random lattice displacements in the corresponding lamella.

From the above, it can be seen that the determination of $2 N_{l}(\sim 100-200)$ values of strain and damage is reduced to the determination of $N_{w}{ }^{e}+N_{w}{ }^{D W}(\sim 10-30) B$-spline weights. With the RaDMAX program, these parameters are determined by fitting the calculated curve to the observed data using either the classical Levenberg-Marquardt least-squares fitting algorithms (local optimization, fast) or a generalized simulated annealing, GSA, algorithm (global optimization, slow) (Boulle \& Debelle, 2010). Guess strain/damage profiles can be imported from complementary measurements and manually optimized through the graphical interface before the fitting procedure.

The RaDMaX program is written using the Python programming language (www.python.org) and the graphical user interface has been developed with the wxPython toolkit (www.wxpython.org). The NumPy library (www.numpy.org) has been used to benefit from vector programming capabilities and hence increased computing performances (van de Walt, 2011). The SciPy library (www.scipy.org) provided advanced numerical and mathematical functions like random number generation, interpolation, fast convolution algorithms and least-squares fitting algorithms (Oliphant, 2007). Finally, the Matplotlib plotting library (www.matplotlib.org) has been used for all plots in the interface. With the present implementation the computation of a typical XRD curve is performed in a few tens of milliseconds.

\section{Program description and usage}

Screenshots of the graphical user interface of the RaDMaX program are displayed in Fig. 1 and Fig. 2. In this section we provide details on each element of the interface and give guidelines for an optimal usage of the program. 


\subsection{The File menu}

This menu is the necessary first step in the use of the program as it allows to load experimental

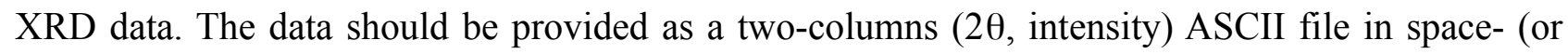
tab-) separated format. The $2 \theta$ values have to be equally spaced (constant step). For the moment RaDMaX can only handle data recorded in symmetric coplanar geometry (i.e. the conventional $\theta$ $2 \theta$ scan), as this is the most commonly used geometry in the analysis of irradiated materials.

Additional entries in the File menu allow to import strain/damage depth profiles. Similarly to the XRD data, these profiles should be provided as a two-columns ASCII file with the depth below the surface (in Angstroms) as the first column. These profiles are automatically fitted with Eq. 1 and the corresponding $B$-spline weights are extracted from the fit (and stored in *.txt files when the session is saved). The corresponding curves are then displayed in the corresponding window. The two last entries in the menu allow to save a session, or to load a previously saved session. Saving a session results in writing all necessary parameters in a text file (*.ini) that can be manually modified if needed.

\subsection{Main window}

The main window is separated in four distinct sections: experiment (Fig. 1a), material (Fig. 1b), strain/damage (Fig. 1c) and plot (Fig. 1d) sections.

The experiment section gathers all informations regarding the XRD measurement: the X-ray wavelength (in Angstroms), the width (full-width at half-maximum, in $2 \theta$ degrees) and shape (between 0 and 1) of the resolution function of the diffractometer and the background noise (normalized to the maximum intensity). The resolution function of the diffractometer is an essential aspect of the fitting procedure as it affects the width and shape of all features of the diffraction curve (the interference fringes as well as the Bragg peak from the virgin region of the crystal). The width and shape of the resolution function can be determined by the measurement of a virgin 
sample. The background noise is usually low and depends on the type of detector and optics used. Because the angular range spanned by the data is in general relatively narrow, the background noise is assumed to be a constant that is added to the calculated profile.

The material section contains all information related to the nature and structure of the material, as well as the Miller indices of the reflection considered. The nature of the crystal is selected from a drop-down menu that lists the existing structures. These structures are given in the form of text files, located in the "structures" folder, that contain the name of the elements and the atomic coordinates. Users can hence easily add and use their own structure files. A structure file is organized as follows, for example in the case of cubic $\mathrm{ZrO}_{2}$ :

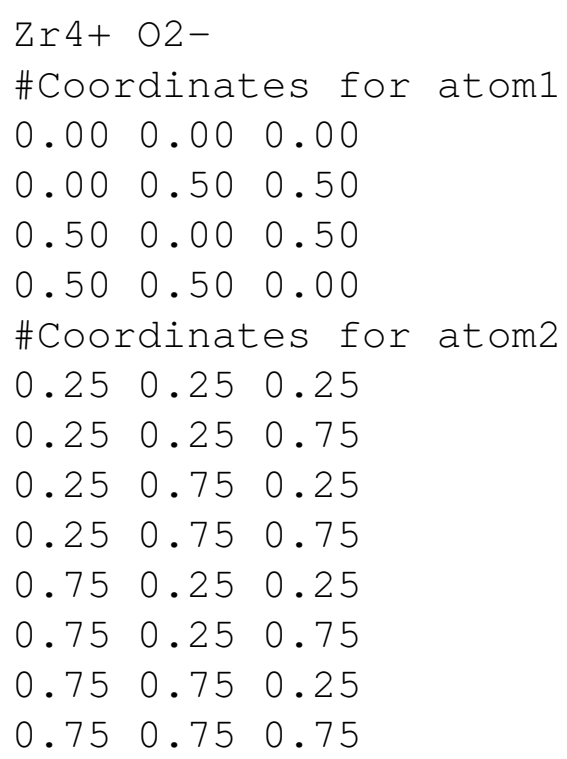

The structure factor is computed from these coordinates. The atomic scattering factors are computed according to the method of Waasmaier \& Kirfel (1995), and absorption and anomalous dispersion are included in the calculation using the Brennan-Cowan database (Brennan \& Cowan, 1992).

The strain/damage section allows to define and parametrize the models used to describe the strain and damage profiles. The irradiated layer can be chosen to have either an abrupt or a smooth (default option) interface with the virgin crystal. In the latter case, three $B$-splines weights (equal to 0 for the strain and to 1 for the damage) are added to those defined by the user, to ensure a gradual transition from the irradiated region to the underlying virgin crystal. 
The number of $B$-splines weights, $N_{w}{ }^{e}$ and $N_{w}{ }^{D W}$, can be defined in the corresponding fields. There is no general rule for the choice of these parameters and each case must be carefully tested. Typical values are in the 5-15 range. Lower values result in smooth curves and faster fitting, but the model might be unable to render fine details of the strain/damage profiles. Conversely, higher values are necessary to describe subtle features of the strain/damage profiles but may lead to unphysical oscillations in the fitted profiles and in increased computing times. The Min./Max. fields are the acceptable variation ranges of the $B$-spline weights during the GSA fitting.

The thickness of the irradiated region and number of lamellas $N_{l}$ (required for the computation of the XRD curve) are also defined in this section. As mentioned earlier, the number of lamellas has a crucial influence on both the computing time and the accuracy of the computed curve. A simple rule to determine this parameter is, starting from a relatively low value, to progressively increase its value so as to remove all spurious oscillations in the computed curve, especially in the low intensity regions. The smallest value of $N_{l}$ yielding a flawless curve corresponds to the best accuracy/speed compromise. Typical values are in the 50-100 range and should be multiples of both $N_{w}{ }^{e}$ and $N_{w}{ }^{D W}$.

In the plot section are represented the strain and damage depth profiles and the corresponding computed XRD curve superimposed on the experimental data. The strain/damage profiles are either plotted with default values of the $B$-spline weights $(=1)$, or using the values imported from guess strain/damage profiles. The strain/damage profiles are interactive in the sense that the user can modify their shape by locally dragging the curve via control points represented as circles in the plots (this action actually corresponds to changing the $B$-spline weights defined earlier). The overall curve can be scaled up or down by pressing the " $u$ " key and using the mouse wheel. The corresponding XRD curve is updated in real time for an easy evaluation of the quality of the simulation. This step allows to adjust the guess strain/profile before moving to the automatic fitting procedure. 


\subsection{Fitting window}

In this window (Fig. 2), it is possible to select the fitting algorithm (either GSA or LevenbergMarquardt). GSA should be used only when the guess strain/damage profiles fail to approximately describe the XRD data, or when there are no guess profiles. GSA is a global search algorithm which randomly explores the parameters space and is therefore relatively slow as compared to local optimization algorithms. On the other hand, the conventional least-squares algorithm is fast but requires a rather good estimation of the starting strain/damage profiles to be refined. In this latter case, it must be borne in mind that the resulting fitted profiles may exhibit unphysical values, as for instance, DW larger than unity. When using GSA, the parameters are constrained to lie in the range defined in the previous window (a feature not implemented in the Levenberg-Marquardt algorithm we use). Both algorithms can be used successively to progressively refine the solution.

The parameters described below are only relevant to the GSA algorithm. Within this algorithm, the parameters perform random jumps in the parameters space (bounded by the Min./Max. values provided by the user). The length of the jumps is parametrized by an artificial temperature which is progressively lowered during the procedure. High temperatures $(\sim 1000)$ correspond to high "thermal motion" and long jumps are possible. Conversely, at low temperature $(\sim 1)$ the system is almost frozen and the parameters can only performed short jumps, which actually corresponds to a local search mechanism. The temperature is decreased in (cooling) steps to ensure that at each temperature, the parameters have been sufficiently varied. The algorithm stops when the predefined number of cycles is reached. Alternatively, the fit can be aborted at any moment by the user. The additional parameters $\left(q_{a}, q_{v}\right.$ and $\left.q_{T}\right)$ allow to modify the behavior of the GSA algorithm. The default values ensure an optimal functioning of the algorithm. Further details regarding this algorithm can be found in Boulle \& Debelle (2010).

After each fitting cycle the calculated XRD curve and the corresponding strain and damage depth profiles are stored in ASCII files located in the same folder as the XRD data. The quality of the fit, 
measured using the residual error [equation (2) in Boulle \& Debelle (2010)], is provided at the end of each fitting cycle.

\subsection{Comments and examples}

The main idea behind RaDMaX is to overcome the "phase problem" which originates from the fact that lattice displacements affect the phase of the diffracted amplitude, whereas the quantity measured in practice is the intensity (where the phase is lost). Nonetheless, the phase shifts induced by lattice displacements give rise to interference features from which the displacement profiles can be retrieved. An important consequence is that only XRD data with visible interference fringes can be used to reliably determine the strain/damage profiles.

In all the cases we investigated these last years (Boulle \& Debelle, 2010; Debelle, Channagiri et al., 2014; Debelle, Boulle et al., 2014), the strain profiles were found to be extremely robust against sample alignment errors or sample quality: slightly misaligned samples, or even polycrystalline materials were found to give reliable and reproducible results regarding the strain profile (provided that a good fit could be obtained). On the contrary, the damage profiles were found to be extremely sensitive to sample alignment or sample quality. Any deviation of the diffracted intensity from its nominal value (due to a tilt of the crystal, or to crystals with finite lateral dimensions and irregular shapes) leads to unphysical values of the DW factor (i.e. DW $>1$ ).

Finally, in order to keep the computing time as low as possible, the convolution of the intrinsic diffraction profile with the resolution function is performed using a fast convolution algorithm. For optimal results, the data should be sampled in such a way that in the narrowest feature before convolution (in general the diffraction peak emanating from the virgin crystal) there should be at least three data points above the half-maximum to describe the peak shape. In general this condition is easily fulfilled except in the case of relatively light elements (such as in SiC for instance), where special care should be brought to data sampling. A too weak sampling rate will mainly results in a 
wrong DW profile.

In the last five years, the approach implemented in RaDMaX has been applied to several cases, and examples can be found in our previous publications (Boulle \& Debelle, 2010; Debelle, Channagiri et al., 2014; Debelle, Boulle et al., 2014). Fig. 1 and Fig. 3 show the examples of $300 \mathrm{keV}$ Cs irradiated yttria stabilized zirconia (YSZ, with ion fluence $=3.75 \times 10^{13}$ ions $/ \mathrm{cm}^{2}$ ) and $100 \mathrm{keV} \mathrm{Fe} \mathrm{-}$ irradiated cubic silicon carbide $\left(3 \mathrm{C}-\mathrm{SiC}\right.$, with ion fluence $=4 \times 10^{13}$ ions $\left./ \mathrm{cm}^{2}\right)$. In both cases, the fits are close to perfect. All the associated parameters are given in the figures and the corresponding files can be found in the "examples" folder of the program. The case of YSZ is fairly simple and 6 B-spline functions, for both the strain and damage profiles, were sufficient to achieve a perfect fit. The data has been recorded on a high-resolution laboratory diffractometer. The irradiated region extends over $200 \mathrm{~nm}$ with a maximum strain of $0.55 \%$ located around $50 \mathrm{~nm}$ below the surface, the profile being relatively flat between 15 and $80 \mathrm{~nm}$. The damage is relatively weak with a DW factor of $\sim 0.8$ in the entire irradiated region. The case of $3 \mathrm{C}-\mathrm{SiC}$ is more complicated, partly because of the very low intensity emanating from the irradiated region $\left(10^{4}\right.$ weaker than the main Bragg peak); this data has been recorded at the BM2 beamline at the ESRF (Grenoble, France). Up to 15 splines were necessary here, especially to correctly describe the low strain region (i.e. the intensity close to the main Bragg peak). The strain profile appears more peaked than YSZ (with a maximum of 1.15\% around $30 \mathrm{~nm}$ ). Additionally, the region extending $80 \mathrm{~nm}$ below the surface is heavily damaged, with a very low DW factor of 0.1 .

Finally, it is worth mentioning that since irradiated single crystals resemble simple film/substrate systems, the present program can be straightforwardly adapted to study irradiated (or even only defective) films grown on substrates. It is also possible to consider its adaptation for the analysis of irradiated multilayers. In this case, however, special care has to be taken to correctly take into account irradiation-induced roughening and intermixing that may occur at interfaces as these features are not implemented for the moment. Regarding this last point, we recall that the source 
code is available and users may adapt it to fit their particular needs.

\section{Availability and installation}

The program is distributed under the CeCILL license, a French license similar to and compatible with the General Public License. The program can be downloaded from the GitHub code hosting website: http://aboulle.github.io/RaDMaX.

RaDMaX has been successfully tested on Windows 7 and Windows 10, and different Linux distributions (Debian 8 and Ubuntu 15.04). RaDMaX requires the following programs to be installed on the computer: python 2.7, SciPy, MatPlotLib and wxPython.

\section{References}

Averback, R. S. (1994). J. Nucl. Mater. 216, 49-62.

Bartels, W. J., Hornstra., J., Lobeek, D. J. W. (1986) Acta. Cryst. A 42, 539-545.

Boulle, A., Masson, O., Guinebretière, R., Dauger, A. (2003). J. Appl. Cryst. 36, 1424-1431.

Boulle, A. Guinebretière, R., Dauger, A. (2003). J. Phys. D.: Appl. Phys 38, 3907-3920.

Boulle, A., Conchon, F., Guinebretière, R. (2009). J. Appl. Cryst. 42, 85-92.

Boulle, A., Debelle, A. (2010). J. Appl. Cryst. 43, 1046-1052.

Brennan, S., Cowan, P. L. (1992). Rev. Sci. Instr. 63, 850-853.

Debelle, A., Channagiri, J., Thomé, L., Décamps, B., Boulle, A., Moll, S., Garrido, F., Behar, M., Jagielski, J. (2014). J. Appl. Phys. 115, 183504.

Debelle, A., Boulle, A., Chartier, A., Gao, F., Weber, W. J. (2014). Phys. Rev. B 90, 174112.

Dhara, S. (2007). Crit. Rev. Sol. State Mater. Sci. 32, 1-5.

Elliman, R. G., Williams, J. S. (2015). Curr. Opin. Sol. State Mater. Sci. 19, 49-67.

Klappe, J. G. E. \& Fewster, P. F. (1994). J. Appl. Cryst. 27, 103-110.

Milita, S. \& Servidori, M. (1995). J. Appl. Cryst. 28, 666-672. 
Oliphant, T. E. (2007). Comput. Sci. Eng. 9, 10-20.

Pietsch, U., Holý, V., Baumbach, T. (2004). High-Resolution X-ray Scattering - From Thin Films to Lateral Nanostructures. New York: Springer-Verlag.

Rieutord, F., Mazen, F., Reboh, S., Penot, J. D., Bilteanu, L., Crocombette, J. P., Vales, V., Holy, V., Capello, L. (2013). J. Appl. Phys. 113, 153511.

Speriosu, V. S. (1981). J. Appl. Phys. 52, 6094-6103.

Takagi, S. (1969) J. Phys. Soc. Jpn 26, 1239-1253.

Taupin, D. (1964) Bull. Soc. Franç. Minér. Crist. 87, 469-511.

Thomé, L., Garrido, F. (2001). Vacuum 63, 619-626.

van de Walt, S., Colbert, S. C., Varoquaux, G. (2011). Comput. Sci. Eng. 13, 22-30.

Waasmaier, D., Kirfel, A. (1995). Acta Crystallogr. A 51, 416-431.

Weber, W. J. (2000). Nucl. Inst. Meth. Phys. Res. B 166-167, 98-106.

Zaumseil, P., Winter, U., Cembali, F., Servidori, M., Sourek, Z. (1987). Phys. Stat. Sol. (a) 100, 95104. 


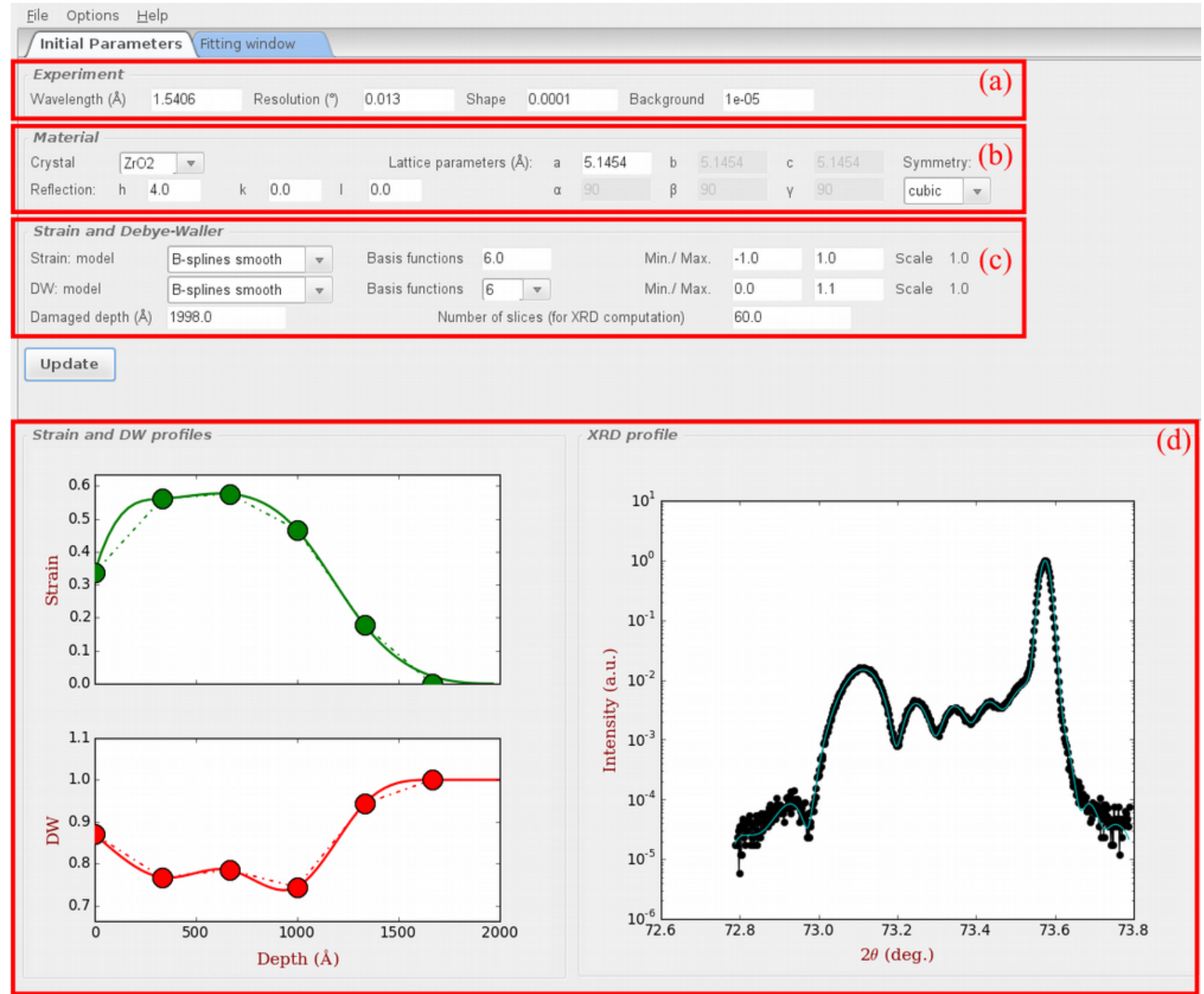

Fig. 1. Screenshot of RaDMaX running in Kubuntu 15.04. The simulation corresponds to irradiated yttria - stabilized zirconia. 


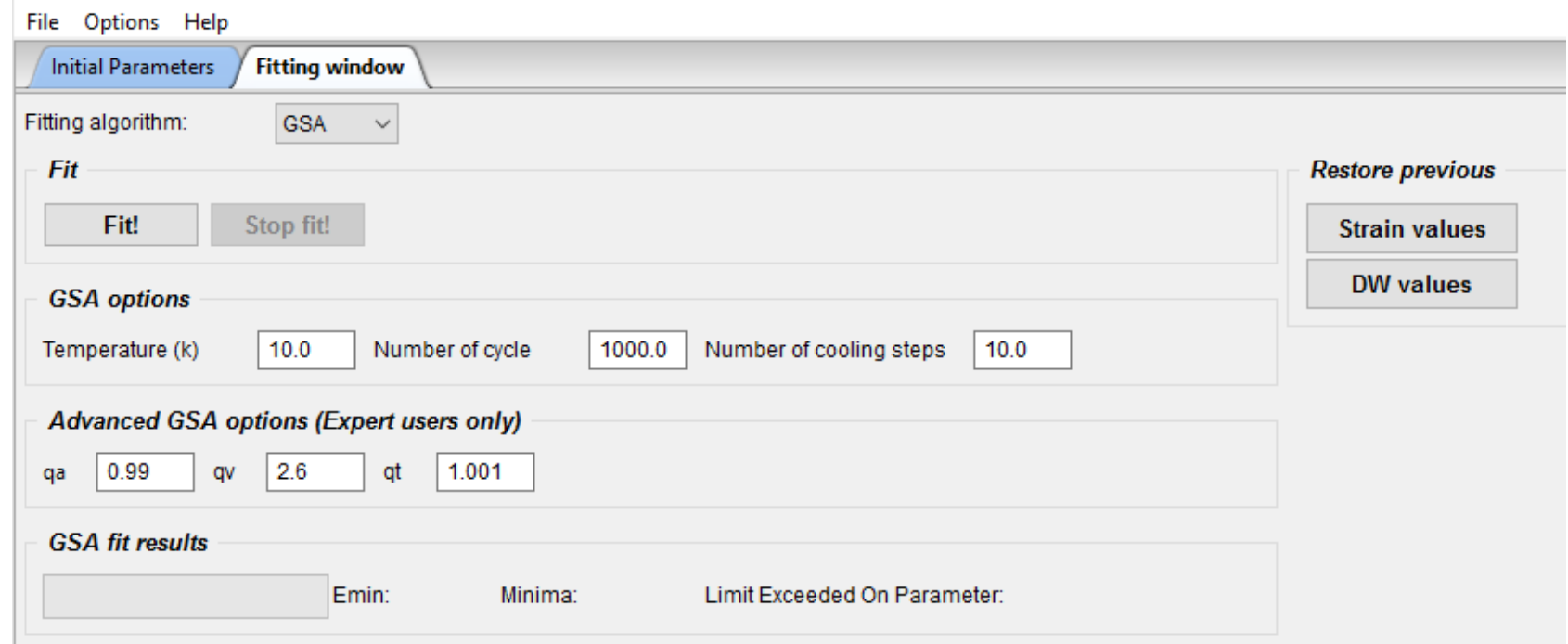

Fig. 2. Screenshot of the fitting window. 


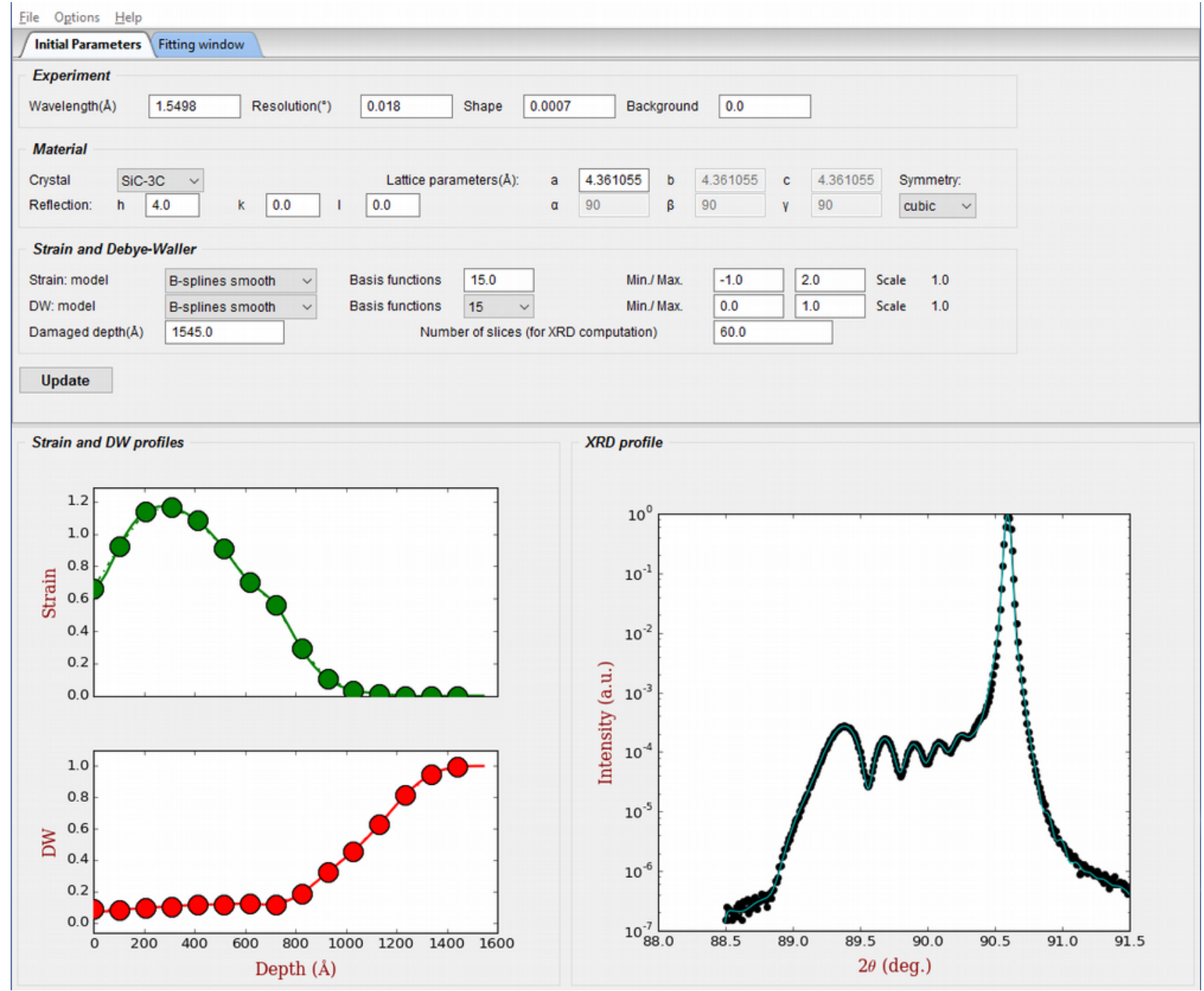

Fig. 3. Screenshot of RaDMaX running in Windows 10. The simulation corresponds to irradiated silicon carbide. 